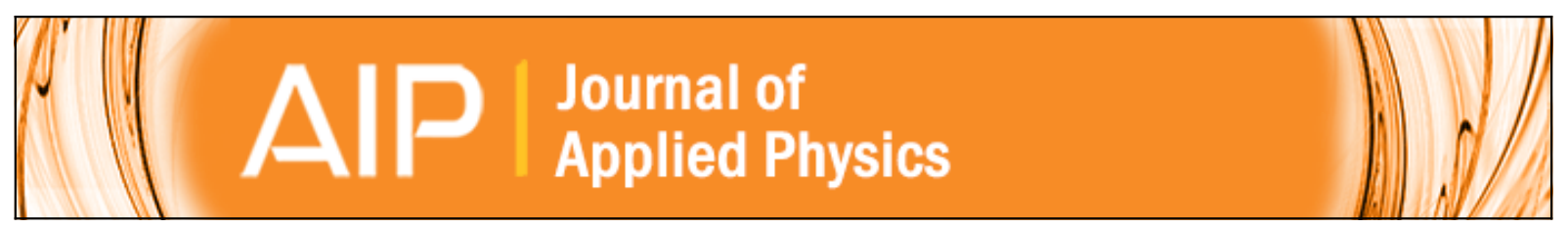

\title{
A unified approach to modelling the charge state of monatomic hydrogen and other defects in crystalline silicon
}

Chang Sun, Fiacre E. Rougieux, and Daniel Macdonald

Citation: Journal of Applied Physics 117, 045702 (2015); doi: 10.1063/1.4906465

View online: http://dx.doi.org/10.1063/1.4906465

View Table of Contents: http://scitation.aip.org/content/aip/journal/jap/117/4?ver=pdfcov

Published by the AIP Publishing

\section{Articles you may be interested in}

Electrical levels in nickel doped silicon

J. Appl. Phys. 116, 173704 (2014); 10.1063/1.4901003

A unified physical model of Seebeck coefficient in amorphous oxide semiconductor thin-film transistors

J. Appl. Phys. 116, 104502 (2014); 10.1063/1.4895046

Recombination in compensated crystalline silicon for solar cells

J. Appl. Phys. 109, 043704 (2011); 10.1063/1.3555588

Recombination activity of interstitial iron and other transition metal point defects in $\mathrm{p}$ - and $\mathrm{n}$-type crystalline silicon

Appl. Phys. Lett. 85, 4061 (2004); 10.1063/1.1812833

Deep level of iron-hydrogen complex in silicon

J. Appl. Phys. 82, 3828 (1997); 10.1063/1.365746

Frustrated by

old technology?

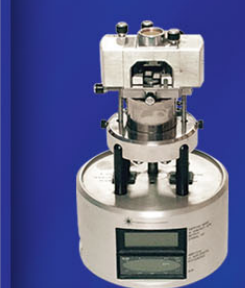

Is your AFM dead

and can't be repaired?

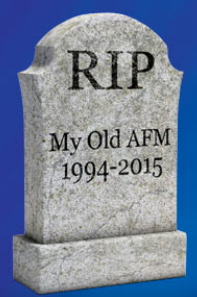

Sick of bad customer support?

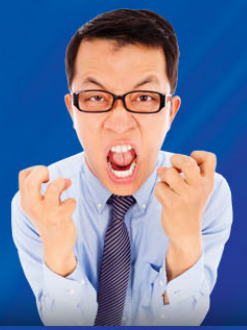

It is time to upgrade your AFM Minimum $\$ 20,000$ trade-in discount for purchases before August 31st

Asylum Research is today's technology leader in AFM

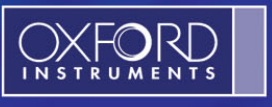




\title{
A unified approach to modelling the charge state of monatomic hydrogen and other defects in crystalline silicon
}

\author{
Chang Sun, ${ }^{\text {a) }}$ Fiacre E. Rougieux, and Daniel Macdonald \\ Research School of Engineering, College of Engineering and Computer Science, \\ The Australian National University, Canberra, ACT 0200, Australia
}

(Received 26 November 2014; accepted 12 January 2015; published online 22 January 2015)

\begin{abstract}
There are a number of existing models for estimating the charge states of defects in silicon. In order of increasing complexity, these are (a) the Fermi-Dirac distribution, (b) the Shockley-Last model, (c) the Shockley-Read-Hall model, and (d) the Sah-Shockley model. In this work, we demonstrate their consistency with the general occupancy ratio $\alpha$, and show that this parameter can be universally applied to predict the charge states of both monovalent and multivalent deep levels, under either thermal equilibrium or steady-state conditions with carrier injection. The capture cross section ratio is shown to play an important role in determining the charge state under nonequilibrium conditions. The application of the general occupancy ratio is compared with the quasi-Fermi levels, which are sometimes used to predict the charge states in the literature, and the conditions where the latter can be a good approximation are identified. The general approach is then applied to the prediction of the temperature- and injection level-dependent charge states for the technologically important case of multivalent monatomic hydrogen, and several other key monovalent deep levels including $\mathrm{Fe}, \mathrm{Cr}$, and the boron-oxygen complex in silicon solar cells. For the case of hydrogen, we adapt the model of Herring et al., which describes the charge states of hydrogen in thermal equilibrium, and generalize it for non-equilibrium conditions via the inclusion of the general occupancy ratio, while retaining the pre-factors which make the model more complete. Based on these results, the impact of temperature and injection on the hydrogenation of the key monovalent defects, and other pairing reactions, are discussed, demonstrating that the presented model provides a rigorous methodology for understanding the impact of charge states. (C) 2015 AIP Publishing LLC. [http://dx.doi.org/10.1063/1.4906465]
\end{abstract}

\section{INTRODUCTION}

Hydrogen can bind with and passivate a wide range of defects and impurities in crystalline silicon, such as grain boundaries, dislocations, shallow dopants, and also many point-like deep levels. ${ }^{1,2}$ Such hydrogenation, by reducing the recombination activity of deep defects, can be of crucial importance in silicon devices, especially solar cells. The charge state of monatomic hydrogen plays a key role in this passivation process, because differently charged species of hydrogen have dramatically different diffusivities, and in principle, will only bond with defects in suitable charge states. ${ }^{3-5}$ In this work, we will describe and apply a rigorous approach to calculating the fraction of monatomic hydrogen in various charge states as a function of both temperature and injection level. The same approach will also be applied to some key monovalent deep-level defects in silicon solar cells, namely, interstitial $\mathrm{Fe}\left(\mathrm{Fe}_{\mathrm{i}}\right), \mathrm{Cr}_{\mathrm{i}}$ and the boron-oxygen (BO) complex. Based on these results, we will discuss the potential for these key defects to be passivated by the oppositely charged hydrogen in silicon, and identify the temperature ranges and injection levels that are likely to result in the greatest degree of hydrogenation.

\section{BACKGROUND}

\section{A. Previous charge state-prediction models}

In the simplest case of a monovalent defect in thermal equilibrium, the Fermi level $E_{F}$ and the spin degeneracy of the defect may be used to determine the occupancy probability of the state. ${ }^{6,7}$ When neglecting degeneracies, the result is simply the Fermi-Dirac distribution. For the more complex case of a multivalent defect in thermal equilibrium, Shockley and Last ${ }^{8}$ proposed a model (referred to here as the ShockleyLast model) to predict the charge distribution as a function of the Fermi level and temperature. An important concept in their work is the ratio of the occupancy probability by holes to that by electrons for each energy level introduced by the defect, which, of course, also determines the concentration ratios of the various charge states of the defect. As shown by Shockley and Last, when neglecting degeneracies, the occupancy ratio for an energy level introduced by a multivalent defect in thermal equilibrium is identical to that calculated for a monovalent defect with the same energy level. ${ }^{8}$

Whilst the Fermi level model and the Shockley-Last model accurately predict the charge state in thermal equilibrium, they do not apply to the important case of nonequilibrium conditions, which prevail, for example, when annealing under illumination or with an applied bias. In the literature, the quasi-Fermi levels are sometimes used as an approximation to account for the effect of injection in the

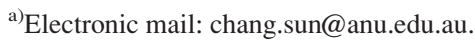


calculation of the charge state of defects. For example, Landsberg studied the case where the deviation from thermal equilibrium is very small. ${ }^{9}$ Besides the electron quasi-Fermi level $E_{F c}$ and the hole quasi-Fermi level $E_{F v}$, another quasiFermi level $E_{F t}$ was introduced in his work, which was found to describe the carriers trapped at the defect level very well. ${ }^{9}$ Kimerling et al. used the electron quasi-Fermi level to estimate the charge state of isolated $\mathrm{Fe}_{\mathrm{i}}$ in $\mathrm{p}$-Si, providing an explanation for why FeB pairs can be dissociated by minority carrier injection: namely, that $E_{F C}$ is shifted above the energy level of $\mathrm{Fe}_{\mathrm{i}}$ under injection, leaving this donor level in a neutral charge state, which prohibits the re-pairing process during injection. ${ }^{10}$ Conzelmann et al. applied a similar approach for $\mathrm{Cr}_{\mathrm{i}}$ to explain why $\mathrm{CrB}$ pairs in $\mathrm{p}-\mathrm{Si}$ cannot be dissociated by injection. ${ }^{11}$ However, the quasi-Fermi levels were originally introduced to describe the carrier distributions in the conduction band and the valence band, as the carriers in each band are in quasi-equilibrium with themselves, whereas the two bands are not in a unified equilibrium with one another. ${ }^{12}$ This means, generally, one should not use the quasi-Fermi levels to describe the carrier distribution in the non-equilibrium bandgap. Especially at intermediate or highinjection levels, which commonly occur during the operation of devices such as silicon solar cells, applying the quasiFermi levels to estimate the charge distribution of a defect may generate misleading results.

The case of non-equilibrium steady-state recombination statistics through a monovalent defect was first considered by Shockley and Read, ${ }^{13}$ and Hall ${ }^{14}$ (SRH statistics). This model also gives a more rigorous answer to the problem mentioned above. As shown in their model, the occupancy probability of a defect level in non-equilibrium steady-state depends on not only the carrier concentrations (which are implied by the quasi-Fermi levels) but also the capture and emission rates of the defect level for both electrons and holes. On the basis of these previous works, Sah and Shockley ${ }^{15}$ proposed a rigorous model (referred to here as the Sah-Shockley model), which describes the charge distribution and recombination statistics on multivalent defects in non-equilibrium steady-state. This model was subsequently applied to multivalent defects in silicon including substitutional $\mathrm{Au}\left(\mathrm{Au}_{\mathrm{s}}\right)^{16}$ and $\mathrm{Zn}_{\mathrm{s}}{ }^{17}$

These four models-(a) the Fermi-Dirac distribution (monovalent, thermal equilibrium), (b) the Shockley-Last model (multivalent, thermal equilibrium), (c) the SRH model (monovalent, non-equilibrium), and (d) the Sah-Shockley model (multivalent, non-equilibrium) - represent a set of increasingly complex models that may be applied to determine the charge states of defects in silicon under steady-state conditions. In this work, we will identify the conditions under which these models are accurate, and in particular, compare the non-equilibrium models with the simpler quasiFermi level model to identify the conditions where the latter can be used as a good approximation. The relevant models will then be applied to determine the charge state distribution for the technologically important case of monatomic hydrogen (a multivalent defect), and several other key monovalent deep levels in silicon solar cells, which are thought to be passivated by hydrogen.

\section{B. Charge states of monatomic hydrogen and several key defects}

Monatomic hydrogen is an important and widely studied multivalent defect in silicon. It has been identified as a "negative-U" defect, both theoretically via first-principles calculations, and also experimentally. ${ }^{5,18,19}$ Monatomic hydrogen $\left(\mathrm{H}^{\mathrm{s}}\right.$, where $s$ stands for a specific charge state) can assume three charge states: neutral $\left(\mathrm{H}^{0}\right)$, positive $\left(\mathrm{H}^{+}\right)$, and negative $\left(\mathrm{H}^{-}\right)$. In a comprehensive model, Herring et al. calculated the thermal-equilibrium concentration ratios of various charged monatomic hydrogen, molecular hydrogen, and hydrogen-dopant pairs in silicon. ${ }^{18}$ Their results for monatomic hydrogen can be explained by the Shockley-Last model very well, which we will discuss in more detail in the modelling procedure. Applying the model of Herring et al., Hallam et al. estimated the fractional concentrations of the three charge states of monatomic hydrogen in $\mathrm{p}$-Si under non-equilibrium conditions. ${ }^{20}$ To account for the minority carrier injection, the electron quasi-Fermi level was used in the equations replacing the Fermi level, ${ }^{20}$ which is essentially a simple extension of the model of Herring et al. As discussed above, using the quasi-Fermi levels may, in principle, generate inaccurate results, especially at intermediate and high-injection levels. Applying the more complete Sah-Shockley model should provide a more rigorous way to calculating the charge distribution of hydrogen in silicon in non-equilibrium steady-state, however, this has not been done previously.

What also has not been studied with this more rigorous approach is the charge state of $\mathrm{Fe}_{\mathrm{i}}$ and $\mathrm{Cr}_{\mathrm{i}}$ in silicon under injection. Here, we shall apply the SRH statistics to these two monovalent defects to predict their charge states, and, by combining with the results of the Sah-Shockley model for multivalent hydrogen, discuss the potential for hydrogenation of these defects. In addition, the BO defect and the permanent deactivation of this defect have been investigated in detail in recent years. ${ }^{20-26}$ Some authors have suggested that the permanent deactivation process, which occurs during moderate temperature annealing with simultaneous injection, is in fact caused by charge-state driven hydrogenation of the defect. ${ }^{25-27}$ This metastable defect is known to introduce a donor level localized in the upper band-gap of silicon. ${ }^{28,29}$ Courtesy of this finding, we also calculate the charge state of the BO complex and discuss its potential to bind with hydrogen during annealing and under injection.

\section{MODELLING PROCEDURE}

\section{A. Previous models and the deduced occupancy ratio $\alpha$}

Full details of the four models reviewed above can be found in the literature. ${ }^{6-8,13-15}$ For our current purposes, it is sufficient to discuss and compare them in terms of a single key parameter, the occupancy ratio. This parameter can be used to describe the charge distribution of mono or multivalent defects in thermal equilibrium or non-equilibrium steady state conditions. For unambiguous application to both monovalent and multivalent defects, we define the occupancy ratio 
as follows: following the notations in the Sah-Shockley model, each energy level $E(s+1 / 2)$ in the band gap can assume two charge states: $s$ (when the level is occupied by a hole) and $s+1$ (when occupied by an electron). The occupancy ratio is then simply the ratio of the number $N_{s}$ of defects in charge state $s$ to the number $N_{s+1}$ in charge state $s+1 .^{8,15}$ Here, we neglect degeneracies in the general model, as is often assumed for deep levels as a simplification. ${ }^{30}$

\section{The Fermi-level, the Shockley-Last model, and the thermal occupancy ratio}

Shockley and Last $^{8}$ showed the consistency of their model with the Fermi-Dirac distribution: the occupancy ratio for an energy level $E(s+1 / 2)$ introduced by a monovalent or a multivalent defect in thermal equilibrium can both be expressed by

$$
N_{s} / N_{s+1}=\exp \left[\frac{E\left(s+\frac{1}{2}\right)-E_{F}}{k_{B} T}\right] .
$$

We denote this thermal occupancy ratio as $\alpha_{\text {thermal }}$. It can also be expressed in the form

$$
\alpha_{\text {thermal }}=\frac{p_{0}}{p_{1}}=\frac{n_{1}}{n_{0}},
$$

where $n_{0}$ and $p_{0}$ are the thermal-equilibrium electron and hole densities, and $n_{1}, p_{1}$ are the equilibrium electron and hole densities when the defect energy level coincides with the Fermi level.

\section{SRH model and the general occupancy ratio}

As suggested by the SRH model, ${ }^{13}$ in steady state, the fraction of a monovalent defect occupied by electrons is

$$
f_{t}=\frac{c_{n} \cdot n+c_{p} \cdot p_{1}}{c_{n} \cdot\left(n+n_{1}\right)+c_{p}\left(p+p_{1}\right)},
$$

where $c_{n}$ and $c_{p}$ are the electron and hole capture constants, and $n$ and $p$ are the densities of electrons in the conduction band and holes in the valence band, respectively. The relationship between $c_{n}, c_{p}$ and the electron and hole emission constants $e_{n}$ and $e_{p}$ is

$$
n_{1}=e_{n} / c_{n}, \quad p_{1}=e_{p} / c_{p} .
$$

Using the electron and hole capture cross sections $\sigma_{n}, \sigma_{p}$ and the carrier thermal velocities $v_{\text {th }}$ to replace $c_{n}$ and $c_{p}$, and introducing the capture cross section ratio $k=\sigma_{n} / \sigma_{p}$, Eq. (3) can be reduced to

$$
f_{t}=\frac{1}{1+\frac{k n_{1}+p}{k n+p_{1}}}
$$

The occupancy ratio $\alpha$ can be then obtained,

$$
\alpha=\frac{k n_{1}+p}{k n+p_{1}} .
$$

Note that the ratio $\alpha$ contains information of (a) the densities of carriers $n$ and $p$, (b) the location of the defect level $n_{1}$ and $p_{1}$, and (c) the capture cross section ratio $k$ of the defect level. The first of these specifies the non-equilibrium conditions, and the next two the properties of the defect level. In thermal equilibrium where

$$
\Delta n=\Delta p=0, \quad n=n_{0}, p=p_{0},
$$

the general occupancy ratio simplifies to the thermal occupancy ratio as

$$
\alpha=\frac{k n_{1}+p_{0}}{k n_{0}+p_{1}}=\frac{p_{0}}{p_{1}}=\alpha_{\text {thermal }},
$$

where we have used the fact that $n_{0} p_{0}=n_{1} p_{1}$. What is notable here is that the capture cross section ratio $k$ does not appear in the expression for $\alpha_{\text {thermal }}$.

\section{The Sah-Shockley model and its consistency with the general occupancy ratio}

The occupancy ratio for an energy level $E(s+1 / 2)$ introduced by a multivalent defect given in the more general Sah-Shockley model is in the form of Eq. (9), ${ }^{15}$

$$
\frac{N_{s}}{N_{s+1}}=\frac{c_{p}\left[p+p^{*}\left(s+\frac{1}{2}\right)\right]}{c_{n}\left[n+n^{*}\left(s+\frac{1}{2}\right)\right]},
$$

where $n^{*}(s+1 / 2)$ and $p^{*}(s+1 / 2)$ are defined as

$$
n^{*}\left(s+\frac{1}{2}\right)=e_{p} / c_{n}, \quad p^{*}\left(s+\frac{1}{2}\right)=e_{n} / c_{p} .
$$

Combining Eqs. (4) and (10), we can see that these two parameters are related to $n_{1}$ and $p_{1}$ by

$$
\frac{p^{*}\left(s+\frac{1}{2}\right)}{n_{1}}=\frac{p_{1}}{n^{*}\left(s+\frac{1}{2}\right)}=\frac{c_{n}}{c_{p}}=k .
$$

Using $n_{1}, p_{1}$, and $k$ to replace $n^{*}(s+1 / 2)$ and $p^{*}(s+1 / 2)$ in Eq. (9), the occupancy ratio reverts back to the form in Eq. (6) again.

This demonstrates the consistency of the four models when it comes to the prediction of the charge state. As a general rule, the occupancy ratio of an energy level $E(s+1 / 2)$, whether introduced by a monovalent or a multivalent defect, in thermal equilibrium or in non-equilibrium steady-state, can all be expressed by the parameter $\alpha$ given by Eq. (6).

\section{Comparison of the occupancy ratio and quasi-Fermi level approximations}

As mentioned above, the electron quasi-Fermi level is sometimes used to estimate the charge states of defects under injection in the literature. ${ }^{9-11,20}$ In this section, we compare the general expression for the occupancy ratio $\alpha$ (Eq. (6)), 
with both the electron and hole quasi-Fermi levels approximations. Accordingly, two analogous ratios $\alpha_{c}$ and $\alpha_{v}$, which are associated with $E_{F c}$ and $E_{F v}$, respectively, can be derived,

$$
\begin{aligned}
& \alpha_{c}=\exp \left(\frac{E\left(s+\frac{1}{2}\right)-E_{F c}}{k_{0} T}\right)=\frac{n_{1}}{n}, \\
& \alpha_{v}=\exp \left(\frac{E\left(s+\frac{1}{2}\right)-E_{F v}}{k_{0} T}\right)=\frac{p}{p_{1}} .
\end{aligned}
$$

We note again that $\alpha_{c}$ and $\alpha_{v}$ do not contain the capture cross section ratio $k$. At low injection levels approaching thermal equilibrium, $k$ becomes less important in $\alpha$, and all of these three ratios approach $\alpha_{\text {thermal }}$ as given by Eq. (2).

We also observe that for monovalent defects having a $k$ value larger than unity, and whose energy level is close enough to the conduction band, the electron quasi-Fermi level is a good approximation before the injection becomes too high. The reason for this is that such a defect level has a large value of $n_{1}$, and a negligible value of $p_{1}$ in comparison with the carrier densities $n$ and $p$. At low injection levels, both of $\alpha$ and $\alpha_{c}$ approach $\alpha_{\text {thermal }}$; when the injection level becomes higher, the conditions satisfy $k n_{1}+p \approx k n_{1}$ and $k n+p_{1} \approx k n$, and thus $\alpha \approx \alpha_{c}$. Some examples of this case will be given in Sec. IV below. Similarly, the hole quasiFermi level should be a good approximation for an energy level, which is close enough to the valence band and has $k<1$.

At very high injection levels, where $\Delta n \gg n_{0}, p_{0}, k n_{1}, p_{1}$,

$$
\alpha_{\text {high-injection }}=\frac{1}{k} \text {. }
$$

This equation reveals that at high injection levels, there are abundant carriers in both the conduction and the valence bands, so the carrier distribution at the energy level only depends on its relative ability to capture electrons and holes. Except for the cases where $k$ has a very large value $\left(\alpha_{\text {high-injection }} \approx \alpha_{c} \rightarrow 0\right)$ or a very small value $\left(\alpha_{\text {high-injection }} \approx \alpha_{v} \rightarrow+\infty\right)$, both $\alpha_{c}$ and $\alpha_{v}$ will fail to predict accurately the occupancy ratio $\alpha_{\text {high-injection, }}$, as the essential information from the capture cross section ratio $k$ is missing.

\section{B. Model of the charge distribution of monatomic hydrogen in silicon}

In general, hydrogen in silicon can exist as monatomic hydrogen $\left(\mathrm{H}^{\mathrm{s}}\right)$, molecular hydrogen $\left(\mathrm{H}_{2}\right)$, diatomic hydrogen complexes $\left(\mathrm{H}_{2}{ }^{*}\right)$, or bound to a dangling bond at a defect site. ${ }^{1}$ The equilibrium between those species has been studied by several authors. ${ }^{1,3,18,27}$ According to their findings, the potential energies of $\mathrm{H}_{2}$ and $\mathrm{H}_{2} *$ are lower than those of the monatomic species, but as the reaction from the monatomic species to $\mathrm{H}_{2}$ and $\mathrm{H}_{2}$ * is exothermic, higher temperatures will generally favour the monatomic states more than lower temperatures. ${ }^{1,3,31}$ As higher temperatures can also break some of the bonds between hydrogen and other impurities, ${ }^{1,3,18}$ the net effect of elevating the temperature should be an increase in the concentration of the monatomic hydrogen $N_{H}$, assuming that the total amount of hydrogen in the sample remains fixed. In this work, we will therefore regard the total hydrogen concentration as constant and independent of temperature, and the monatomic hydrogen concentration $N_{H}$ as a variable, which is dependent on the temperature according to some balance with other hydrogen species. Our focus will therefore be on predicting the fractions of different charge states within the unspecified monatomic hydrogen concentration $N_{H}$. Note that these fractions will not depend on the magnitude of $N_{H}$, but the absolute concentrations of $\mathrm{H}^{0}, \mathrm{H}^{+}$, and $\mathrm{H}^{-}$will depend on both $N_{H}$ and the predicted charge fractions.

Courtesy of the findings by first principles calculations, the spin degeneracy of $\mathrm{H}^{\mathrm{s}}$ is included in the model. According to the model of Herring et al., the concentration ratios of $\mathrm{H}^{0}, \mathrm{H}^{+}$, and $\mathrm{H}^{-}$in thermal equilibrium are

$$
\begin{aligned}
& \frac{n_{+}}{n_{0}}=\frac{v_{+} Z_{+}}{v_{0} Z_{0}} \exp \left(\frac{E_{D}-E_{F}}{k_{0} T}\right), \\
& \frac{n_{0}}{n_{-}}=\frac{v_{0} Z_{0}}{v_{-} Z_{-}} \exp \left(\frac{E_{A}-E_{F}}{k_{0} T}\right),
\end{aligned}
$$

where $n_{s}, v_{s}$, and $Z_{s}$ are the concentration, the number of possible sites or orientations per unit cell, and the effective partition function of the corresponding charge state $\mathrm{H}^{\mathrm{s}} . E_{D}$ and $E_{A}$ are the donor level and the acceptor level introduced by monatomic hydrogen. As the temperature dependence of $Z_{s}$ is unknown, we follow the low-temperature approximation $Z_{s}(\mathrm{~T} \rightarrow 0)=1$ suggested in the model of Herring et al. Also, we assume that the energy levels $E_{D}$ and $E_{A}$ are temperature-independent. The values of $v_{s}, E_{D}$ and $E_{A}$ are listed in Table I. ${ }^{18}$

It is evident that Eqs. (15) and (16) are equivalent to applying the definition of the occupancy ratio to the hydrogen levels $E_{D}(+/ 0)$ and $E_{A}(0 /-)$ in thermal equilibrium, but including the effects of the spin degeneracy (included in $v_{s}$ ) and the partition function. As stated in the model of Herring et al., the partition function takes account of the modification which each unit of the species makes in the vibrational partition function of the host plus impurity atoms. ${ }^{18}$ This makes the model of Herring et al. more complete than ShockleyLast model for the case of hydrogen. When neglecting degeneracies and following the low-temperature approximation $Z_{s}=1$, the two exponential terms are exactly those associated with the simple Fermi-Dirac distribution, in agreement with the general Shockley-Last model. To include the nonequilibrium steady-state conditions in the model, we use the occupancy ratios $\alpha^{E_{D}}$ and $\alpha^{E_{A}}$ in place of the exponential terms in Eqs. (15) and (16), which gives

$$
\frac{n_{+}}{n_{0}}=\left(\frac{v_{+} Z_{+}}{v_{0} Z_{0}}\right) \cdot \alpha^{E_{D}}
$$

TABLE I. Values of $v_{s}, E_{D}$, and $E_{A}$ in the monatomic hydrogen model.

\begin{tabular}{ccccc}
\hline \hline$v_{0}$ & $v_{+}$ & $v_{-}$ & $E_{D}$ & $E_{A}$ \\
\hline 8 & 4 & 2 & $E_{c}-0.16 \mathrm{eV}$ & $\left(E_{c}+E_{v}\right) / 2-0.064 \mathrm{eV}$ \\
\hline \hline
\end{tabular}




$$
\frac{n_{0}}{n_{-}}=\left(\frac{v_{0} Z_{0}}{v_{-} Z_{-}}\right) \cdot \alpha^{E_{A}} .
$$

Equations (17) and (18) enable us to predict the fractions of $\mathrm{H}^{0}, \mathrm{H}^{+}$, and $\mathrm{H}^{-}$as the temperature and the injection level vary. Note that our modification of the model of Herring et al. is essentially the inclusion of the occupancy ratio to account for non-equilibrium conditions, whilst retaining the parameters $v_{s}$ and $Z_{s}$ of the model of Herring et al. However, this requires knowledge of the capture cross section ratios $k^{E_{D}}$ and $k^{E_{A}}$. Unfortunately, these are not reported in the literature for the defect levels of monatomic hydrogen. However, as monatomic hydrogen is a point-like impurity in silicon, in this work we estimate these capture cross section ratios by assuming that $k^{E_{D}}$ and $k^{E_{A}}$ follow the observed trend in the capture cross section ratios of point-like transition-metal impurities in silicon. Macdonald and Geerligs showed that for point-like transition-metal impurities in silicon, including $\mathrm{Ti}, \mathrm{V}, \mathrm{Cr}, \mathrm{Mo}, \mathrm{Fe}, \mathrm{Au}$, and $\mathrm{Zn}$, a comparison of energy levels with the same charge states revealed that the deeper levels have greater $k$ values, and that these values follow a quite regular trend. ${ }^{32}$ Following these observations, for donor levels $(+/ 0)$, the hydrogen donor level $\left(E_{c}-0.16 \mathrm{eV}\right)$ is shallower than any of $\mathrm{Cr}_{\mathrm{i}}\left(E_{c}-0.22 \mathrm{eV}\right), \mathrm{Ti}_{\mathrm{i}}\left(E_{c}-0.27 \mathrm{eV}\right)$, $\mathrm{Mo}_{\mathrm{i}}\left(E_{v}+0.28 \mathrm{eV}\right)$, and $\mathrm{Fe}_{\mathrm{i}}\left(E_{v}+0.38 \mathrm{eV}\right)$. For acceptor levels $(0 /-)$, the hydrogen acceptor level $\left(E_{v}+0.496 \mathrm{eV}\right)$ is a little shallower than the level $E_{c}-0.55 \mathrm{eV}$ introduced by $\mathrm{Au}_{\mathrm{s}}$, but deeper than the $\mathrm{Zn}_{\mathrm{s}}$ level at $E_{v}+0.33 \mathrm{eV}{ }^{32}$ Also bearing in mind that donor levels generally have $k$ values larger than unity, ${ }^{32}$ we arrive at the following estimations:

$$
\begin{aligned}
& 1<k^{E_{D}}<k^{\mathrm{Cr}}=3.2, \quad k^{E_{D}} \approx 1 \\
& 0.34=k^{\mathrm{Zn}}>k^{E_{A}}>k^{\mathrm{Au}}=0.02, \quad k^{E_{A}} \approx k^{\mathrm{Au}}, \quad k^{E_{A}} \approx 0.05 .
\end{aligned}
$$

The value of $k^{C r}$ is from Ref. 33, while other values conform with those in Ref. 32. Note that in any case, the value of $k^{E_{D}}$ should not have a significant impact on the results before the injection level becomes high, as the hydrogen donor level is very close to the conduction band, as discussed above.

\section{RESULTS AND DISCUSSIONS}

\section{A. Two examples $\left(\mathrm{Fe}_{\mathrm{i}}\right.$ and $\left.\mathrm{Cr}_{\mathrm{i}}\right)$ comparing the general model with quasi-Fermi level approximations}

Here, we compare the general occupancy ratio $\alpha$ and two analogous ratios $\alpha_{c}$ and $\alpha_{v}$ derived from the quasi-Fermi level approximations, for the specific cases of $\mathrm{Fe}_{\mathrm{i}}$ and $\mathrm{Cr}_{\mathrm{i}}$. Note that although we only show the results for $\mathrm{p}-\mathrm{Si}$ in this section, similar analyses apply for n-type silicon.

Figure 1 shows the injection-dependent occupancy probability (the probability to be occupied by an electron) of the $\mathrm{Fe}_{\mathrm{i}}$ level in $\mathrm{p}$-Si calculated with $\alpha, \alpha_{c}$, and $\alpha_{v}$ at room temperature. The occupancy probability is related to the occupancy ratio via $f=1 /(1+\alpha)$. The recombination parameters of $\mathrm{Fe}_{\mathrm{i}}$ are taken from Ref. 34. As can be seen by comparing the three curves, neither of the quasi-Fermi levels yields a good approximation for the whole injection range for the $\mathrm{Fe}_{\mathrm{i}}$ level.

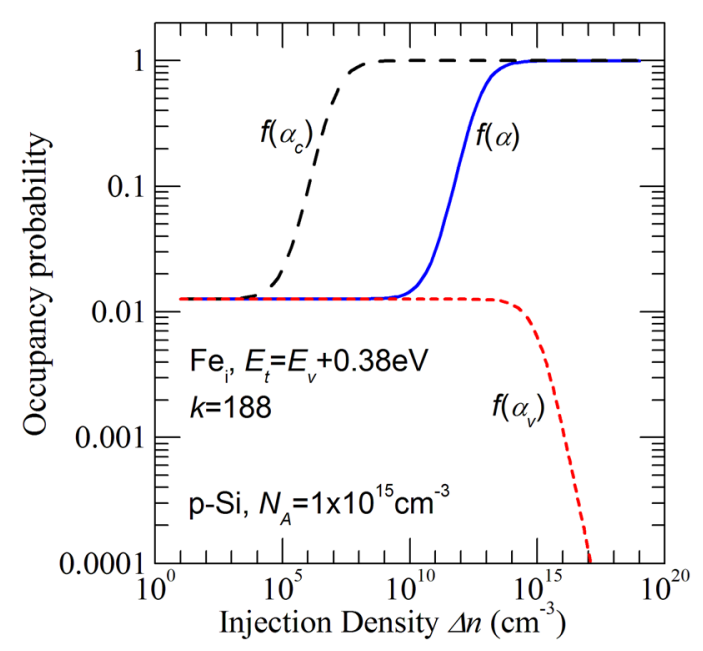

FIG. 1. Occupancy probability of the $\mathrm{Fe}_{\mathrm{i}}$ energy level as a function of the injection density at $300 \mathrm{~K}$ in p-Si with a doping level of $1 \times 10^{15} \mathrm{~cm}^{-3}$. The three curves are calculated with $\alpha_{c}, \alpha_{v}$, and $\alpha$, respectively.

Based on the use of the electron quasi-Fermi level $E_{F c}$ in the calculation, one may expect that all of the isolated $\mathrm{Fe}_{\mathrm{i}}$ atoms are occupied by electrons and become neutral at a very low injection level of about $\Delta n=1 \times 10^{8} \mathrm{~cm}^{-3}$, as shown by the curve $f\left(\alpha_{c}\right)$. This would mean that FeB pairs should not be stable under very low injection levels. However, as predicted with the more rigorous occupancy ratio $\alpha$ shown by the curve $f(\alpha)$, an injection level of about $\Delta n=1 \times 10^{14} \mathrm{~cm}^{-3}$ is required to make all of the isolated $\mathrm{Fe}_{\mathrm{i}}$ neutral. This is consistent with observations of FeB pairs in silicon, which have often been observed to remain paired and stable under moderate injection levels. ${ }^{35}$

Figure 2 shows the injection-dependent occupancy of the $\mathrm{Cr}_{\mathrm{i}}$ level in $\mathrm{p}$-Si calculated with $\alpha, \alpha_{c}$, and $\alpha_{v}$. The recombination parameters of $\mathrm{Cr}_{\mathrm{i}}$ are taken from Ref. 33. As shown by the curve $f(\alpha)$, an injection level around $1 \times 10^{16} \mathrm{~cm}^{-3}$ has to be achieved to obtain a significant fraction of neutral $\mathrm{Cr}_{\mathrm{i}}$, which explains why the injection-stimulated dissociation of $\mathrm{CrB}$ pairs has not been observed before. ${ }^{36,37}$ As discussed in the modelling procedure, we observed that for some

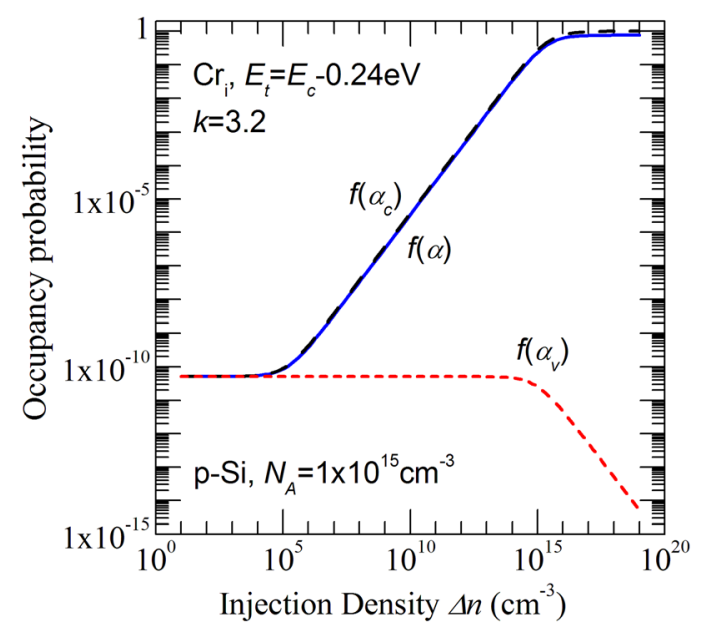

FIG. 2. Occupancy probability of the $\mathrm{Cr}_{\mathrm{i}}$ energy level as a function of the injection density at $300 \mathrm{~K}$ in $\mathrm{p}$-Si with a doping level of $1 \times 10^{15} \mathrm{~cm}^{-3}$. The three curves are calculated with $\alpha_{c}, \alpha_{v}$, and $\alpha$, respectively. 
monovalent defects whose energy levels are located close to the conduction band and with $k>1$, the electron quasi-Fermi level can be a reasonable approximation before the injection becomes high. The $\mathrm{Cr}_{\mathrm{i}}$ level is a good example of such a case, as shown by the coinciding curves $f(\alpha)$ and $f\left(\alpha_{c}\right)$. However, at very high injection levels, $\alpha_{c}$ always approaches 0 and $f\left(\alpha_{c}\right)$ approaches 1 ; but $\alpha$ approaches $1 / k=1 / 3.2$ and $f(\alpha)$ approaches $76 \%$ for $\mathrm{Cr}_{\mathrm{i}}$. That means that even at very high injection levels, only about $76 \%$ of the $\mathrm{Cr}_{\mathrm{i}}$ atoms are in the neutral state.

Furthermore, as the sign of the gradient

$$
\frac{\partial \alpha}{\partial \Delta n}=\frac{k\left(n_{0}-p_{0}\right)-k^{2} n_{1}+p_{1}}{\left(k n_{0}+k \Delta n+p_{1}\right)^{2}}
$$

is injection-independent, the injection dependence of the occupancy ratio as well as the occupancy probability should be monotonic. In $\mathrm{p}$-Si, this term is always negative regardless of the doping level for the $\mathrm{Cr}_{\mathrm{i}}$ level, indicating that $76 \%$ is the upper limit of the fraction of neutral $\mathrm{Cr}_{\mathrm{i}}$ one can achieve in $\mathrm{p}$-Si. By comparison, for $\mathrm{Fe}_{\mathrm{i}}, \alpha$ approximates 0 at high injection levels because $\mathrm{Fe}_{\mathrm{i}}$ has such a large capture cross section ratio (about 580), ${ }^{38}$ meaning that almost all $\mathrm{Fe}_{\mathrm{i}}$ atoms are neutral at high injection levels.

\section{B. Charge states of monovalent defects: $\mathrm{Fe}_{\mathrm{i}}, \mathrm{Cr}_{\mathrm{i}}$, and the BO complex}

Figure 3 shows the modelling results of the temperatureand injection level-dependent $f\left(\mathrm{Fe}_{\mathrm{i}}{ }^{+}\right)$in both $\mathrm{n}$ - and $\mathrm{p}-\mathrm{Si}$ with a doping level of $1 \times 10^{15} \mathrm{~cm}^{-3}$. The band gap narrowing effect at elevated temperatures is included in the calculation, ${ }^{39}$ while the band gap narrowing effect induced by high-injection is not considered, as this effect is not significant up to the injection level of $1 \times 10^{17} \mathrm{~cm}^{-3} \cdot{ }^{40}$ The relative position of the $\mathrm{Fe}_{\mathrm{i}}$ level in the band gap is considered temperature-independent, and this is also assumed for the energy levels of $\mathrm{Cr}_{\mathrm{i}}$, $\mathrm{BO}$ complex and monatomic hydrogen. We show the results at injection levels of $1 \times 10^{13} \mathrm{~cm}^{-3}$, $1 \times 10^{15} \mathrm{~cm}^{-3}$, and $1 \times 10^{17} \mathrm{~cm}^{-3}$ to represent low, intermediate, and high injection conditions, respectively. In thermal equilibrium at a lower temperature range, most of $\mathrm{Fe}_{\mathrm{i}}$ is neutral in $\mathrm{n}-\mathrm{Si}$ and is positive in $\mathrm{p}-\mathrm{Si}$. With the increase of the injection level, $f\left(\mathrm{Fe}_{\mathrm{i}}{ }^{+}\right)$increases in $\mathrm{n}$-Si and decreases in p-Si. At high injection levels, the results in both types of silicon become similar as the doping becomes much lower than the excess carrier densities. Another condition where the doping becomes unimportant is at high temperatures when silicon becomes intrinsic. In this case, the injection also becomes less important, and the occupancy ratio $\alpha$ approaches $\alpha_{\text {thermal }}$ given by Eq. (2).

Figure 4 gives the modelling results for $\mathrm{Cr}_{\mathrm{i}}$. With the results in both types of silicon shown on the same scale, it is clear that the doping becomes unimportant at high injection levels and at high temperatures. In comparison with $\mathrm{Fe}_{i}$, the carrier injection has a much smaller impact on the fraction of $\mathrm{Cr}_{\mathrm{i}}{ }^{+}$: an obvious change in the charge distribution begins at an intermediate injection level of $1 \times 10^{15} \mathrm{~cm}^{-3}$, and in the same injection range, $f\left(\mathrm{Cr}_{\mathrm{i}}{ }^{+}\right)$only varies within one order of magnitude.

Similarly to $\mathrm{Cr}_{\mathrm{i}}$, injection also has a much less significant impact on the charge distribution of the BO complex, which is shown in Figure 5. The calculation is based on the finding that the $\mathrm{BO}$ complex can be associated with a donor level at $E_{c}-0.41 \mathrm{eV}$ having a $k$ value of $9.3 .^{28,29}$ As the BO complex is deactivated at elevated temperatures, ${ }^{22}$ we only calculate the charge state in a temperature range below $300^{\circ} \mathrm{C}$. The permanent deactivation of the BO complex at about $200^{\circ} \mathrm{C}$ under illumination has been observed in $\mathrm{p}-\mathrm{Si},{ }^{23,24}$ and has been attributed to passivation via hydrogenation by some authors, ${ }^{25-27}$ although we note that other models exist which are not based on hydrogenation to explain the regeneration. ${ }^{41,42}$ This reaction also occurs in compensated n-type silicon containing boron, but in this case, the deactivation was observed to be partially unstable under illumination at room temperature by some authors, ${ }^{43}$ although another recent report indicates the deactivation may be stable. ${ }^{44}$

These experimental observations can potentially be explained by the charge state of the BO complex under different conditions. As shown in Figure 5, at $200{ }^{\circ} \mathrm{C}$, there is always a significant fraction of the $\mathrm{BO}$ complex, which is positively charged in both types of silicon, unless the injection becomes too high, ensuring the hydrogen passivation of this defect can proceed. However, at room temperature, the BO complex is mostly neutral in compensated $\mathrm{n}-\mathrm{Si}$, while it
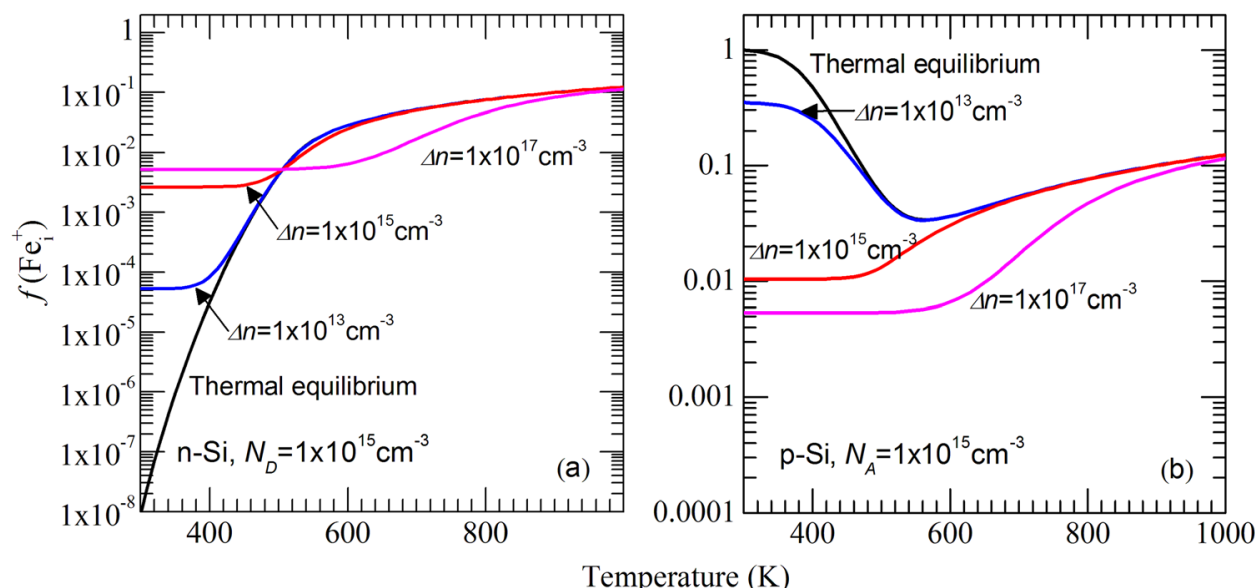

FIG. 3. The fraction of $\mathrm{Fe}_{\mathrm{i}}{ }^{+}$of the total isolated $\mathrm{Fe}_{i}$ as a function of both temperature and injection density $\Delta n$ in (a) $\mathrm{n}$-Si and (b) p-Si with a doping level of $1 \times 10^{15} \mathrm{~cm}^{-3}$.

Temperature (K) 

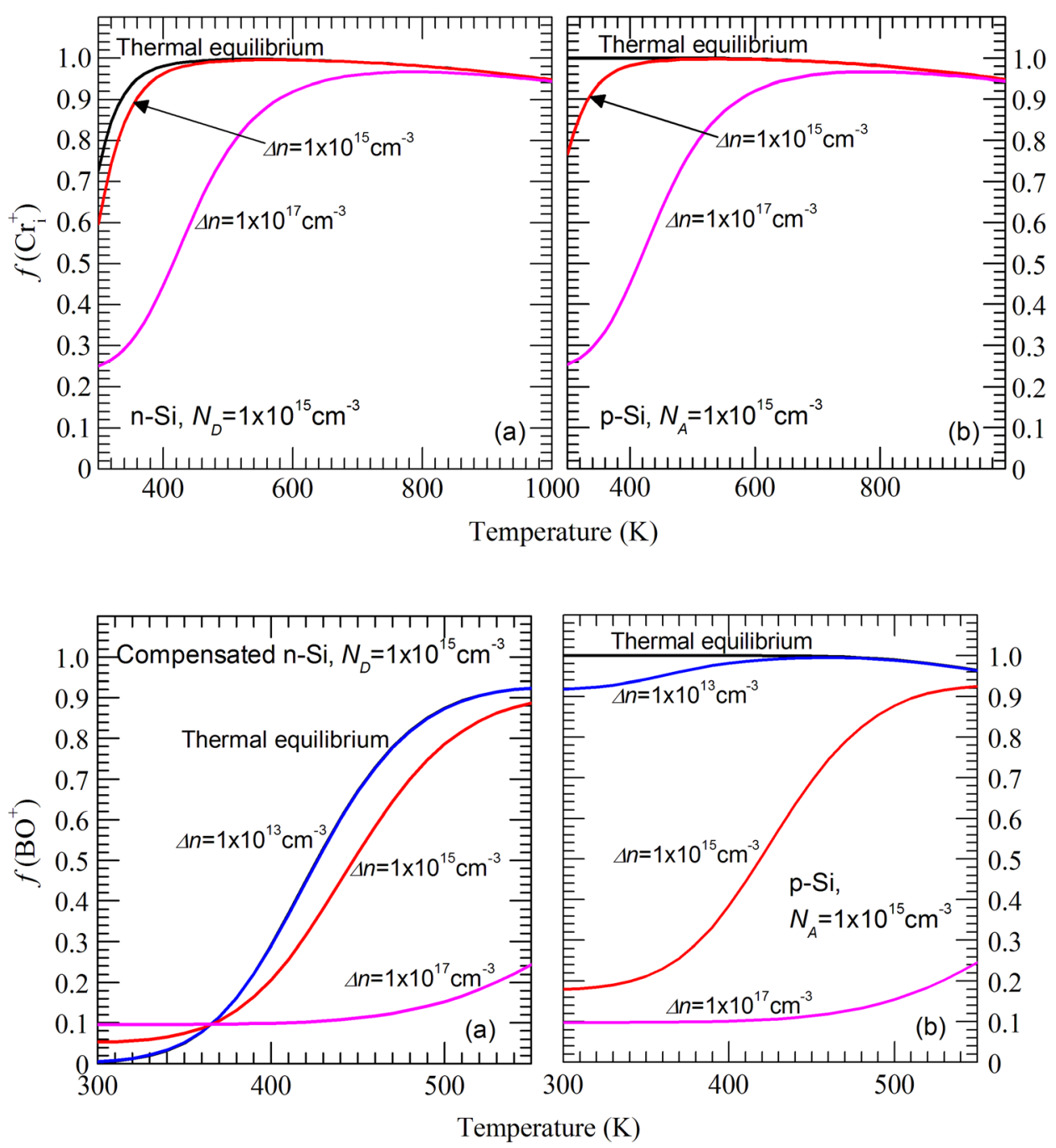

FIG. 4. The fraction of $\mathrm{Cr}_{i}^{+}$of the total isolated $\mathrm{Cr}_{\mathrm{i}}$ as a function of both temperature and injection density $\Delta n$ in (a) $\mathrm{n}$-Si and (b) p-Si with a doping level of $1 \times 10^{15} \mathrm{~cm}^{-3}$. is mostly positively charged in p-Si. In compensated $\mathrm{n}-\mathrm{Si}$, if the bond between the BO complex and hydrogen is broken by illumination, the $\mathrm{BO}$ complex becomes neutral and will not pair with hydrogen again, leading to a reversal of the deactivation process. This will not occur in $\mathrm{p}-\mathrm{Si}$, as the two components of the defect can quickly re-form due to their opposite charge states. This may explain why the deactivation of the BO complex is unstable in compensated n-type silicon.

We note that the case of the $\mathrm{BO}$ defect deactivation in $\mathrm{p}-\mathrm{Si}$ is analogous to the fact that $\mathrm{CrB}$ pairs cannot be broken under illumination in $\mathrm{p}-\mathrm{Si}$, due to the $\mathrm{Cr}_{\mathrm{i}}$ being positively charged, and re-pairing immediately after dissociation. On the other hand, the unstable $\mathrm{BO}$ deactivation in $\mathrm{n}-\mathrm{Si}$ is similar to $\mathrm{FeB}$ pairs in $\mathrm{p}-\mathrm{Si}$, as the $\mathrm{Fe}$ is neutral under injection at room temperature, meaning the pairs cannot re-form.

\section{Charge distribution of monatomic hydrogen}

Figure 6 shows the predicted fractions of $\mathrm{H}^{+}, \mathrm{H}^{-}$, and $\mathrm{H}^{0}$ as a function of temperature and injection level in $\mathrm{n}-\mathrm{Si}$ with a doping of $1 \times 10^{15} \mathrm{~cm}^{-3}$. Figure 7 shows the results in $\mathrm{p}$-Si with the same doping level. The first observation is that in thermal equilibrium at room temperature, $\mathrm{H}^{-}$is the majority species in $\mathrm{n}-\mathrm{Si}$, while $\mathrm{H}^{+}$is the majority species in $\mathrm{p}-\mathrm{Si}$, in agreement with the results of other studies. ${ }^{1,18}$ In the high-temperature intrinsic condition, $\mathrm{H}^{+}$is always the majority charge state as the Fermi level is pinned around the middle of the band gap, which is far below the hydrogen donor level $(+/ 0)$ but very close to the acceptor level $(0 /-)$. Minority carrier injection generally increases $f\left(\mathrm{H}^{+}\right)$and decreases $f\left(\mathrm{H}^{-}\right)$in $\mathrm{n}-\mathrm{Si}$, while its effect is reversed in $\mathrm{p}-\mathrm{Si}$. What is also notable is that, under most circumstances, injection increases $f\left(\mathrm{H}^{0}\right)$ in both $\mathrm{n}$ - and $\mathrm{p}$-Si. The neutral hydrogen atom $\mathrm{H}^{0}$ is recognised to play an important role in hydrogenation in some works, and the reason has been widely attributed to its much higher diffusivity in comparison with other charge states, ${ }^{20,27,45,46}$ although other reports have partly contradicted this. ${ }^{47}$ In addition, in the model suggested by Wilking et al., the permanent deactivation of BO defects is proposed as passivation by $\mathrm{H}^{0}$ atoms. ${ }^{27}$ Thus according to the results, it can be advantageous to have a higher injection level before the hydrogenation process as it helps distribute monatomic hydrogen throughout the silicon wafer from the hydrogen source, which is generally a dielectric layer at the surfaces. ${ }^{20,46}$

At this stage, we shall discuss how our results differ from the simple extension of the model of Herring et al. Since injection becomes unimportant in the temperature range where intrinsic conditions prevail, we only discuss the 


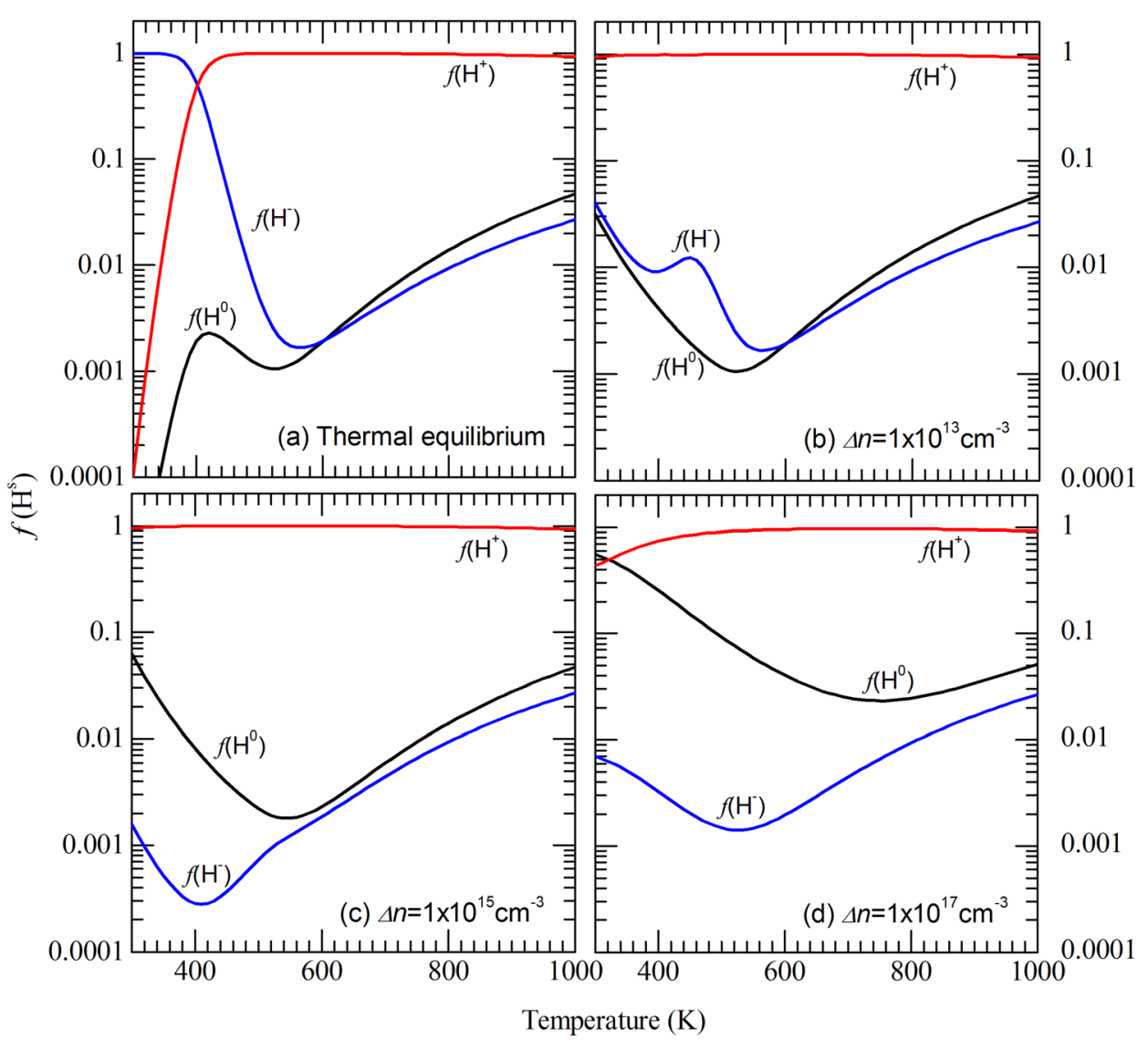

Temperature (K)

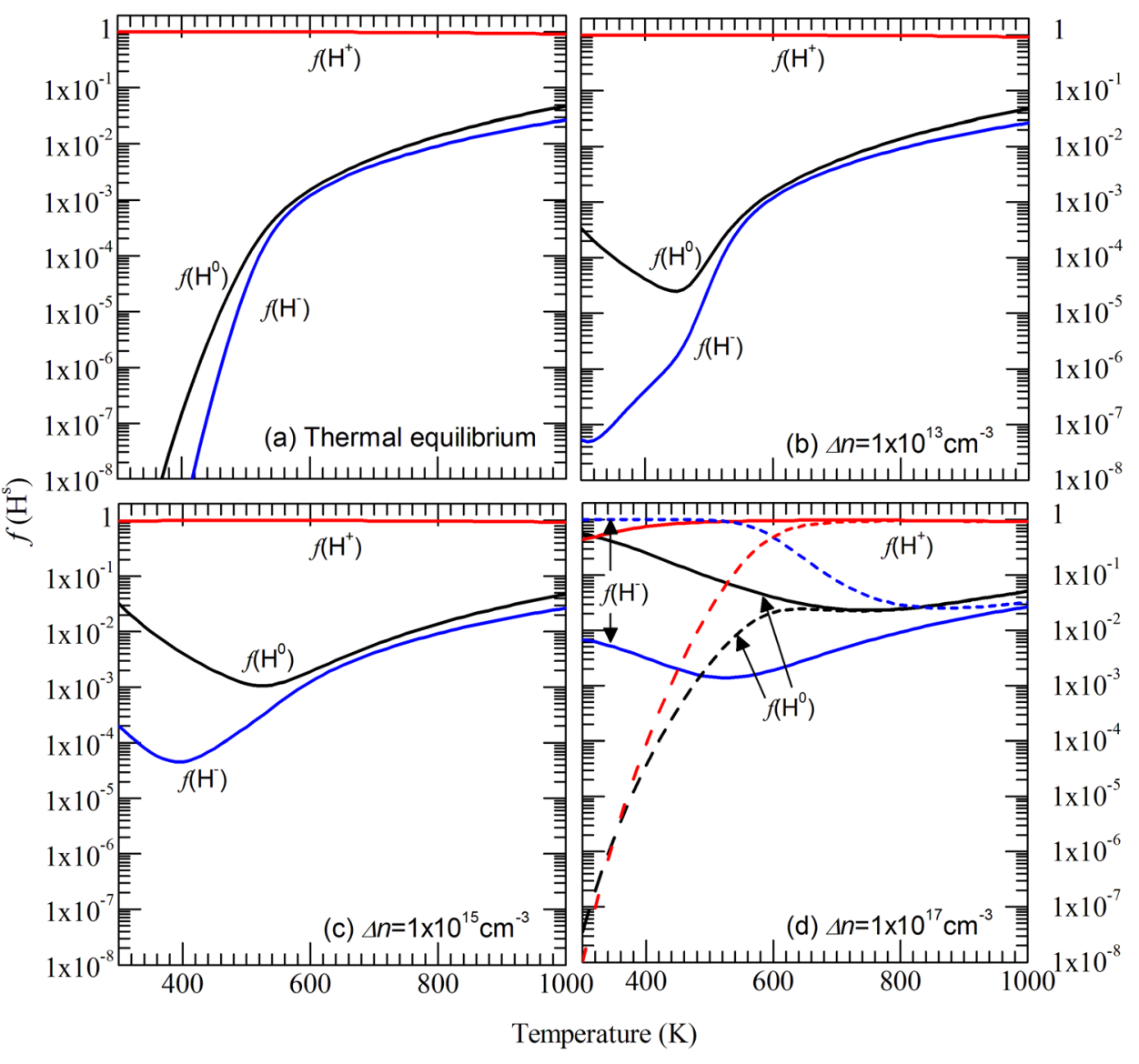

FIG. 6. The fractions of $\mathrm{H}^{+}, \mathrm{H}^{-}$, and $\mathrm{H}^{0}$ of the total monatomic hydrogen as a function of temperature (a) in thermal equilibrium, and with injection densities of (b) $\Delta n=1 \times 10^{13} \mathrm{~cm}^{-3}$, (c) $\Delta n=1 \times 10^{15} \mathrm{~cm}^{-3}$, and (d) $\Delta n=1 \times 10^{17} \mathrm{~cm}^{-3}$ in n-Si with a doping level of $1 \times 10^{15} \mathrm{~cm}^{-3}$.
FIG. 7. The fractions of $\mathrm{H}^{+}, \mathrm{H}^{-}$, and $\mathrm{H}^{0}$ of the total monatomic hydrogen as a function of temperature (a) in thermal equilibrium, and with injection densities of (b) $\Delta n=1 \times 10^{13} \mathrm{~cm}^{-3}$, (c) $\Delta n=1 \times 10^{15} \mathrm{~cm}^{-3}$, and (d) $\Delta n=1 \times 10^{17} \mathrm{~cm}^{-3}$ in p-Si with a doping level of $1 \times 10^{15} \mathrm{~cm}^{-3}$. In addition, the results of the simple extension of the model of Herring et al. are shown in dashed lines for comparison for the injection level of $1 \times 10^{17} \mathrm{~cm}^{-3}$. 
results in the lower temperature range. In thermal equilibrium, both the simple extension of the model of Herring et al. and this model coincide with the original model developed by Herring et al., as expected. ${ }^{18,20}$ However, they diverge significantly as the injection level increases. At high injection levels, $\alpha_{c}$ (in the simple extension of the model of Herring et al.) approaches 0 for both energy levels introduced by monatomic hydrogen, which results in

$$
n_{+} \ll n_{0} \ll n_{-} .
$$

For our model, however, combining Eqs. (17) and (18) and the high injection level approximation Eq. (14), gives

$$
\frac{n_{+}}{n_{0}}=\frac{1}{2 k^{E_{D}}}, \quad \frac{n_{-}}{n_{0}}=\frac{k^{E_{A}}}{4} .
$$

As an acceptor level (0/-) captures electrons when it is neutral and captures holes when it is negatively charged, $\sigma_{n}$ should be generally smaller than $\sigma_{p}$ due to the Coulombic attraction associated with the latter process, resulting in a $k$ value lower than unity for most acceptor levels. ${ }^{32}$ In this respect, $n \_n_{0}$ should be smaller than a quarter according to Eq. (22), which is an obvious difference from the condition expressed by Eq. (21) in the simple extension of the model of Herring et al. We demonstrate this difference in p-Si at an injection level of $\Delta n=1 \times 10^{17} \mathrm{~cm}^{-3}$, shown by Figure 7(d).

\section{Discussion of the hydrogenation of the key defects}

In the process of defect hydrogenation, monatomic hydrogen in a certain charge state is more inclined to bond with oppositely charged defects, or possibly with neutral defects. The key deep-level defects we have discussed in this work, $\mathrm{Fe}_{\mathrm{i}}, \mathrm{Cr}_{\mathrm{i}}$, and the $\mathrm{BO}$ complex, all introduce a single donor level $(+/ 0)$ in the band gap. They should therefore be mostly passivated by negative hydrogen through the reaction $\mathrm{M}^{+}+\mathrm{H}^{-} \rightarrow \mathrm{MH}$, where $\mathrm{M}$ signifies the defect in question. We may assume this as an elementary reaction so that, at a given temperature, the reaction rate linearly depends on the product of the concentrations of $\mathrm{M}^{+}, \mathrm{H}^{-}$and the rate constant $k_{r}$, according to the law of mass action. The rate constant $k_{r}$ is a function of temperature and is governed by the activation energy $E_{a}$ via the Arrhenius equation. The activation energy for the proposed hydrogenation of the $\mathrm{BO}$ complex is found to be about $0.62 \mathrm{eV},{ }^{23}$ and that for the hydrogenation of $\mathrm{Fe}$ is in the $1.2-2 \mathrm{eV}$ range. ${ }^{1,48}$ This reveals a relatively strong temperature dependence of the rate constant. In addition, as discussed in the modelling procedure of hydrogen, higher temperatures are expected to significantly increase the concentration of monatomic hydrogen $N_{H}$ at the expense of molecular hydrogen. As a result, if dehydrogenation at even higher temperatures is avoided, higher temperatures are expected to always accelerate the hydrogenation reaction, potentially outweighing the impact of the changes in $f\left(\mathrm{H}^{-}\right)$and $f\left(\mathrm{M}^{+}\right)$. Thus, we shall focus on the effect of injection on the reaction rate at fixed temperatures, where the modelling results can be interpreted without the complications of the temperature effects, which are not included in the model.
At a fixed temperature, we shall regard the concentration of the defect [M], $k_{r}$, and $N_{H}$ as invariant so that the reaction rate only depends linearly on $f\left(\mathrm{H}^{-}\right) \cdot f\left(\mathrm{M}^{+}\right)$. Figure 8 shows the predicted $f\left(\mathrm{H}^{-}\right) \cdot f\left(\mathrm{M}^{+}\right)$as the injection level varies at $200^{\circ} \mathrm{C}$ in $\mathrm{n}$ - and $\mathrm{p}$-Si. At this temperature, a high fraction of $\mathrm{FeB}$ pairs and $\mathrm{CrB}$ pairs are dissociated and hence the metals are free to bind with $\mathrm{H}$; but a certain injection level is needed to keep the BO complex active through a recombinationenhanced defect formation process. ${ }^{21}$ At higher temperatures, the curves will become flatter over the whole injection range, but will still approach the same values as this temperature at higher injection levels. This is because at higher injection levels, the occupancy ratio approximates the inverse capture cross section ratio, which is temperatureindependent, as expressed by Eq. (14). Also shown in the figures is the fraction of negative hydrogen. Comparing the results shown in Figures 3-7, we can see that the injection has a much more significant impact on $f\left(\mathrm{H}^{-}\right)$than $f\left(\mathrm{M}^{+}\right)$, so the injection-dependence of the product $f\left(\mathrm{H}^{-}\right) \cdot f\left(\mathrm{M}^{+}\right)$is dominated by the injection-dependence of $f\left(\mathrm{H}^{-}\right)$. As a result, as shown by Figure 8, the curves $f\left(\mathrm{H}^{-}\right) \cdot f\left(\mathrm{M}^{+}\right)$all have a similar shape to $f\left(\mathrm{H}^{-}\right)$in each type of silicon.

As shown in the figures, thermal equilibrium conditions, in other words with no injection, should favour the hydrogenation reactions in $\mathrm{n}-\mathrm{Si}$, while in $\mathrm{p}-\mathrm{Si}$, higher injection levels are always preferable to accelerate the reaction; these conditions are exactly those that maximize $f\left(\mathrm{H}^{-}\right)$in each type. The modelling result of $f\left(\mathrm{H}^{-}\right) \cdot f\left(\mathrm{BO}^{+}\right)$in $\mathrm{p}-\mathrm{Si}$ is in agreement with the experimental results showing that a higher injection level results in higher reaction rates and greater amount of hydrogenated $\mathrm{BO}$ defect in $\mathrm{p}-\mathrm{Si}^{24}$

In comparison, acceptor-like defects are mostly passivated by positive hydrogen via the reaction $\mathrm{M}^{-}+\mathrm{H}^{+} \rightarrow \mathrm{MH}$. As shown by Figures 6 and 7, at above $400 \mathrm{~K}$, the fraction of positive hydrogen is nearly injection- and temperatureindependent, always approximating unity. Thus, at a fixed temperature, the injection-dependence of the product $f\left(\mathrm{H}^{+}\right) \cdot f\left(\mathrm{M}^{-}\right)$is dominated by the injection-dependence of $f\left(\mathrm{M}^{-}\right)$. The conditions that favour the hydrogenation reaction the most, therefore, should be the conditions that maximize $f\left(\mathrm{M}^{-}\right)$.

\section{E. Uncertainties in the modelling results}

It should be noted that while the use of the general occupancy factor is in principle rigorous, its application to the cases presented in this work may be uncertain to some degree, due for example, to the following considerations:

(a) In the general model, we neglect degeneracies as a simplification. This will bring uncertainties in the modelling results of $\mathrm{Fe}, \mathrm{Cr}$, and the $\mathrm{BO}$ complex. Note that in the model of monatomic hydrogen, the spin degeneracy is included courtesy of the treatment of Herring et al.

(b) The capture cross section ratios of the defects and the relative positions of the defect levels in the band gap as the band gap narrows are regarded as temperatureindependent, as the temperature-dependence of these parameters is not available in the literature. 


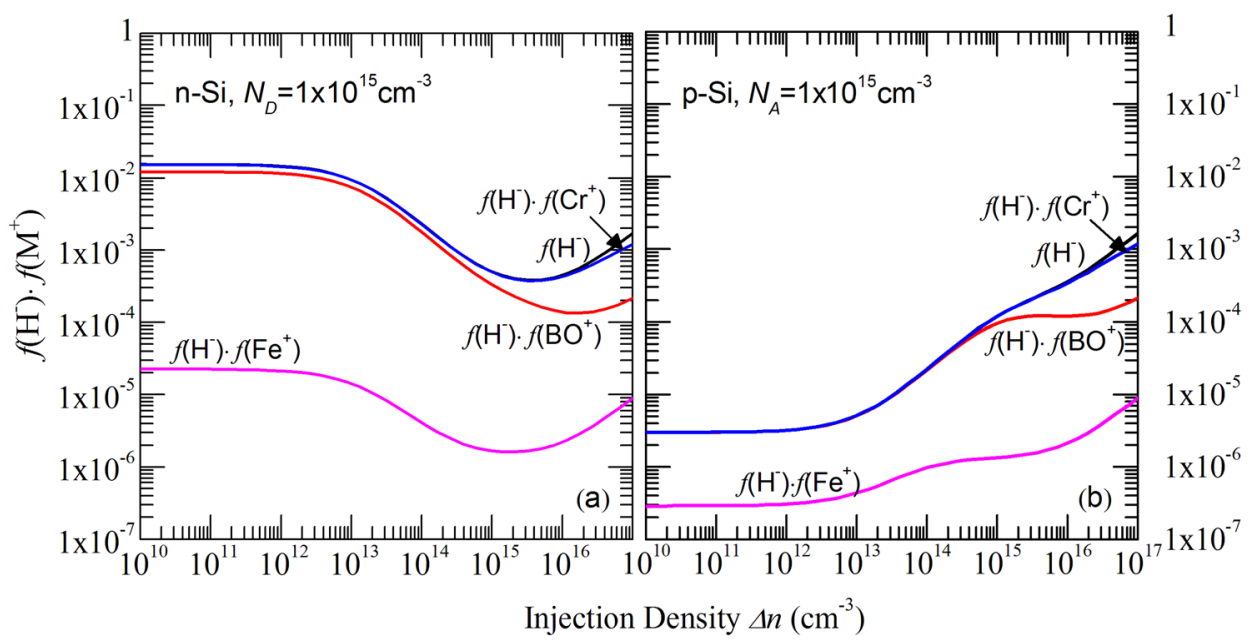

FIG. 8. The product of the fractions of $\mathrm{H}^{-}$and $\mathrm{M}^{+}$, as a function of the injection density at $200^{\circ} \mathrm{C}$ in $\mathrm{n}$ - and $\mathrm{p}$-Si with a doping of $1 \times 10^{15} \mathrm{~cm}^{-3} \cdot f\left(\mathrm{H}^{-}\right)$ is also shown in both figures.

(c) The estimated values of $k^{E_{D}}$ and $k^{E_{A}}$ for the two hydrogen levels are uncertain.

(d) The low-temperature approximation $Z_{s}(\mathrm{~T} \rightarrow 0)=1$ will also add to the uncertainties of the results at elevated temperatures.

\section{CONCLUSIONS}

The four models from the literature (a) the Fermi-Dirac distribution with the spin degeneracy of the defect, (b) the Shockley-Last model, (c) the SRH model, and (d) the Sah-Shockley model, enable the prediction of the charge distribution of deep levels in silicon under various assumptions. We have demonstrated their consistency with the general occupancy ratio $\alpha=\left(k n_{1}+p\right) /\left(k n+p_{1}\right)$, which can be applied to the prediction of the charge distribution of mono/multivalent and in thermal equilibrium/non-equilibrium steady-state conditions. The capture cross section ratio was found to play an important role in determining the charge state under nonequilibrium conditions. This model has been compared with the quasi-Fermi level approximations, and the electron-quasi Fermi level is found to be a good approximation for predicting the occupancy probability of energy levels, which are located close enough to the conduction band and have capture cross section ratios larger than one, for example, the $\mathrm{Cr}_{\mathrm{i}}$ level. However, we have shown that in other cases, the quasi-Fermi level fails to accurately predict the occupancy probability, especially at high injection.

The general model is applied to the prediction of the temperature- and injection level-dependent charge states of several key defects including $\mathrm{Fe}, \mathrm{Cr}$, and the $\mathrm{BO}$ complex in silicon solar cells, and especially monatomic hydrogen. For the case of hydrogen, we have adapted the model of Herring et al., retaining the factors $v_{s}$ and $Z_{s}$, but generalizing it for non-equilibrium via the occupancy ratio from the Sah-Shockley model. For the monovalent deep levels, we have neglected the degeneracies. The presence of carrier injection is found to have a much larger impact on the fraction of negative hydrogen than on those key defects. Based on these results, we have discussed the impact of temperature and injection on the hydrogenation of the key defects. Higher temperatures are very likely to always accelerate the hydrogenation reaction (up to the point when dehydrogenation begins to dominate). However, at a fixed temperature in the lower temperature range, the conditions that will favour hydrogenation of these donor-like defects the most are the conditions that maximize the fraction of negative hydrogen, namely, the thermal equilibrium conditions in $\mathrm{n}-\mathrm{Si}$ and the high-injection conditions in $\mathrm{p}-\mathrm{Si}$, while the conditions that favour hydrogenation of acceptor-like defects are those that maximize the fraction of negatively charged defects. In general, the presented approach provides a rigorous methodology for understanding the impact of charge states on hydrogenation of defects and other pairing reactions in silicon.

\section{ACKNOWLEDGMENTS}

This work has been supported through the Australian Renewable Energy Agency (ARENA) fellowships program, project 1-GER010, and the Australian Centre for Advanced Photovoltaics; and also by the Australian Research Council (ARC) Future Fellowships program.

${ }^{1}$ S. Pearton, J. Corbett, and T. Shi, Appl. Phys. A 43, 153 (1987).

${ }^{2}$ J. I. Hanoka, Hydrogen in Disordered and Amorphous Solids (Springer, 1986), p. 81.

${ }^{3}$ K. Chang and D. Chadi, Phys. Rev. B 40, 11644 (1989).

${ }^{4}$ C. G. Van de Walle, Y. Bar-Yam, and S. Pantelides, Phys. Rev. Lett. 60, 2761 (1988).

${ }^{5}$ C. G. Van de Walle, P. Denteneer, Y. Bar-Yam, and S. Pantelides, Phys. Rev. B 39, 10791 (1989).

${ }^{6}$ P. Landsberg, Proc. Phys. Soc., Sect. A 65, 604 (1952).

${ }^{7}$ P. Landsberg, Proc. Phys. Soc., Sect. A 66, 662 (1953).

${ }^{8}$ W. Shockley and J. Last, Phys. Rev. 107, 392 (1957).

${ }^{9}$ P. Landsberg, Proc. Phys. Soc., Sect. B 70, 282 (1957).

${ }^{10}$ L. Kimerling and J. Benton, Physica B+C 116, 297 (1983).

${ }^{11}$ H. Conzelmann, K. Graff, and E. Weber, Appl. Phys. A 30, 169 (1983).

${ }^{12} \mathrm{P}$. T. Landsberg, Recombination in Semiconductors (Cambridge University Press, 2003).

${ }^{13}$ W. Shockley and W. Read, Jr., Phys. Rev. 87, 835 (1952).

${ }^{14}$ R. N. Hall, Phys. Rev. 87, 387 (1952).

${ }^{15}$ C.-T. Sah and W. Shockley, Phys. Rev. 109, 1103 (1958).

${ }^{16}$ A. Tasch, Jr. and C. Sah, Phys. Rev. B 1, 800 (1970).

${ }^{17}$ C.-T. Sah, P. C. H. Chan, C.-K. Wang, R.-Y. Sah, K. Yamakawa, and R. Lutwack, IEEE Trans. Electron Devices 28, 304 (1981).

${ }^{18}$ C. Herring, N. Johnson, and C. G. Van de Walle, Phys. Rev. B 64, 125209 (2001).

${ }^{19}$ C. G. Van de Walle and J. Neugebauer, Annu. Rev. Mater. Res. 36, 179 (2006). 
${ }^{20}$ B. J. Hallam, P. G. Hamer, S. R. Wenham, M. D. Abbot, A. Sugianto, A. M. Wenham, C. E. Chan, G. Q. Xu, J. Kariem, J. Degoulange, and R. Einhaus, IEEE J. Photovoltaics 4, 88 (2014).

${ }^{21}$ J. Schmidt and K. Bothe, Phys. Rev. B 69, 024107 (2004).

${ }^{22}$ K. Bothe and J. Schmidt, J. Appl. Phys. 99, 013701 (2006).

${ }^{23}$ A. Herguth, G. Schubert, M. Kaes, and G. Hahn, "A new approach to prevent the negative impact of the metastable defect in boron doped cz silicon solar cells," in Conference Record of the IEEE 4th World Conference on Photovoltaic Energy Conversion, Waikoloa, Hawaii, 2006 (IEEE, 2006), p. 940 .

${ }^{24}$ A. Herguth, G. Schubert, M. Käs, and G. Hahn, Prog. Photovoltaics 16, 135 (2008).

${ }^{25}$ G. Krugel, W. Wolke, J. Geilker, S. Rein, and R. Preu, Energy Proc. 8, 47 (2011).

${ }^{26}$ B. J. Hallam, S. R. Wenham, P. G. Hamer, M. D. Abbott, A. Sugianto, C. E. Chan, A. M. Wenham, M. G. Eadie, and G. Xu, Energy Proc. 38, 561 (2013).

${ }^{27}$ S. Wilking, C. Beckh, S. Ebert, A. Herguth, and G. Hahn, Sol. Energy Mater. Sol. Cells 131, 2 (2014).

${ }^{28}$ S. Rein and S. Glunz, Appl. Phys. Lett. 82, 1054 (2003).

${ }^{29}$ J. Adey, R. Jones, D. Palmer, P. Briddon, and S. Öberg, Phys. Rev. Lett. 93, 055504 (2004).

${ }^{30}$ R. F. Pierret and G. W. Neudeck, Advanced Semiconductor Fundamentals (Addison-Wesley Reading, MA, 1987), Vol. 6.

${ }^{31}$ J. Corbett, S. Sahu, T. Shi, and L. Snyder, Phys. Lett. A 93, 303 (1983).

${ }^{32}$ D. Macdonald and L. Geerligs, Appl. Phys. Lett. 85, 4061 (2004).

${ }^{33}$ C. Sun, F. E. Rougieux, and D. Macdonald, J. Appl. Phys. 115, 214907 (2014).
${ }^{34}$ D. Macdonald, J. Tan, and T. Trupke, J. Appl. Phys. 103, 073710 (2008).

${ }^{35}$ L. Geerligs and D. Macdonald, Appl. Phys. Lett. 85, 5227 (2004).

${ }^{36}$ K. Mishra, Appl. Phys. Lett. 68, 3281 (1996).

${ }^{37}$ J. Schmidt, R. Krain, K. Bothe, G. Pensl, and S. Beljakowa, J. Appl. Phys. 102, 123701 (2007).

${ }^{38}$ A. Istratov, H. Hieslmair, and E. Weber, Appl. Phys. A 69, 13 (1999).

${ }^{39}$ M. A. Green, J. Appl. Phys. 67, 2944 (1990).

${ }^{40}$ D. Yan and A. Cuevas, J. Appl. Phys. 114, 044508 (2013).

${ }^{41}$ V. Voronkov, R. Falster, B. Lim, and J. Schmidt, J. Appl. Phys. 112, 113717 (2012).

${ }^{42}$ D. Walter, B. Lim, K. Bothe, V. Voronkov, R. Falster, and J. Schmidt, Appl. Phys. Lett. 104, 042111 (2014).

${ }^{43} \mathrm{~B}$. Lim, "Boron-oxygen-related recombination centers in crystalline silicon and the effects of dopant-compensation," Ph.D. thesis, University of Hannover, 2012.

${ }^{44}$ S. Wilking, J. Engelhardt, S. Ebert, C. Beckh, A. Herguth, and G. Hahn, "High speed regeneration of BO-defects: Improving long term solar cell performance within seconds," in Proceedings of 29th EUPVSEC, Amsterdam 2014, pp. 366-372.

${ }^{45}$ D. Mathiot, Phys. Rev. B 40, 5867 (1989).

${ }^{46} \mathrm{P}$. Hamer, B. Hallam, S. Wenham, and M. Abbott, IEEE J. Photovoltaics 4, 1252 (2014).

${ }^{47}$ R. Rizk, P. De Mierry, D. Ballutaud, M. Aucouturier, and D. Mathiot, Phys. Rev. B 44, 6141 (1991).

${ }^{48} \mathrm{~K}$. McLean, C. Morrow, and D. Macdonald, "Activation energy for the hydrogenation of iron in p-type crystalline silicon wafers," in Conference Record of the IEEE 4th World Conference on Photovoltaic Energy Conversion, Waikoloa, Hawaii, 2006 (IEEE, 2006), p. 1122. 